\title{
ADDENDA
}

\section{Addenda: Octave generalization and the identification of distorted melodies}

\author{
WILLIAM J. HOUSE \\ University of South Carolina \\ Aiken, South Carolina 29801
}

In a recent report (House, 1977), I reported the results of an ANOVA but failed to present the means which were involved in that analysis. This note is prepared to present those data. The undistorted condi-

Table 1

Identification Percentages of Four Melodies by Melodic Distortions

\begin{tabular}{lrrrr}
\hline Treatments & Frere & Twinkle & Yankee & McDonald \\
\hline Undistorted & & & & \\
Low Octave & 71 & 74 & 59 & 90 \\
Middle Octave & 86 & 82 & 63 & 76 \\
High Octave & 90 & 76 & 51 & 74 \\
Random Octave & 35 & 33 & 14 & 25 \\
Alternating & & & & \\
Higher Octave & 23 & 53 & 23 & 23 \\
Lower Octave & 37 & 51 & 12 & 43 \\
Fifth & 6 & 18 & 16 & 10 \\
Fourth & 10 & 8 & 10 & 22 \\
Third & 16 & 12 & 8 & 16 \\
\hline
\end{tabular}

tions were excluded from the analysis, but all distortion treatments are presented in Tables 1 and 2. The mean identification percentage excluding the undistorted conditions are: sine wave, $18.5 \%$; sawtooth wave, $27 \%$; and square wave, $21.7 \%$.

\section{REFERENCE}

House, W. J. Octave generalization and the identification of distorted melodies. Perception \& Psychophysics, 1977, 21. 586.589.

Table 2

Identification Percentages for Three Waveform Conditions by Melodic Distortions

\begin{tabular}{lccc}
\hline Treatments & Sine & Saw & Square \\
\hline Undistorted & & & \\
High Octave & 78 & 71 & 75 \\
Middle Octave & 75 & 81 & 76 \\
Low Octave & 69 & 83 & 72 \\
Random Octave & 23 & 37 & 22 \\
Alternating & & & \\
Higin Octave & 23 & 44 & 25 \\
Low Octave & 27 & 39 & 23 \\
Perfect Fifth & 12 & 7 & 18 \\
Perfect Fourth & 9 & 17 & 15 \\
Major Third & 17 & 18 & 27 \\
\hline
\end{tabular}

